

\title{
Using Path-Dependent Types to Build Type Safe JavaScript Foreign Function Interfaces
} Julien Richard-Foy, Olivier Barais, Jean-Marc Jézéquel

\section{To cite this version:}

Julien Richard-Foy, Olivier Barais, Jean-Marc Jézéquel. Using Path-Dependent Types to Build Type Safe JavaScript Foreign Function Interfaces. ICWE - 14th International Conference on Web Engineering, Jul 2014, Toulouse, France. hal-01026148

\section{HAL Id: hal-01026148 \\ https://hal.inria.fr/hal-01026148}

Submitted on $20 \mathrm{Jul} 2014$

HAL is a multi-disciplinary open access archive for the deposit and dissemination of scientific research documents, whether they are published or not. The documents may come from teaching and research institutions in France or abroad, or from public or private research centers.
L'archive ouverte pluridisciplinaire HAL, est destinée au dépôt et à la diffusion de documents scientifiques de niveau recherche, publiés ou non, émanant des établissements d'enseignement et de recherche français ou étrangers, des laboratoires publics ou privés. 


\title{
Using Path-Dependent Types to Build Type Safe JavaScript Foreign Function Interfaces
}

\author{
Julien Richard-Foy, Olivier Barais, and Jean-Marc Jézéquel \\ IRISA, Université de Rennes, France
}

\begin{abstract}
The popularity of statically typed programming languages compiling to JavaScript shows that there exists a fringe of the programmer population interested in leveraging the benefits of static typing to write Web applications. To be of any use, these languages need to statically expose the Web browser dynamically typed native API, which seems to be a contradiction in terms. Indeed, we observe that existing statically typed languages compiling to JavaScript expose the browser API in ways that either are not type safe, or when they are, typically over constrain the programmers. This article presents new ways to encode the challenging parts of the Web browser API in static type systems such that both type safety and expressive power are preserved. Our first encoding relies on type parameters and can be implemented in most mainstream languages but drags phantom types up to the usage sites. The second encoding does not suffer from this inconvenience but requires the support of dependent types in the language.
\end{abstract}

\section{Introduction}

We recently observed the emergence of several statically typed programming languages compiling to JavaScript (e.g. Java/GWT [10], Dart [9], TypeScript [8], Kotlin $^{1}, \mathrm{Opa}^{2}$, SharpKit ${ }^{3}$, Haxe [2], Scala [7], Idris [1], Elm [6]). Though dynamic typing has its merits and supporters, the mere existence of these statically typed languages shows that there is also a community interested in benefiting from static typing features (allowing e.g. better refactoring support in IDE, earlier error detection, etc.) to write Web applications. Nevertheless, at some point developers need a way to interface with the underlying Web browser dynamically typed native API using a foreign function interface mechanism.

We observe that, even though these languages are statically typed, their integration of the browser API either is not type safe or over constrain the programmers. Indeed, integrating an API designed for a dynamically typed language into a statically typed language can be challenging. For instance, the createElement function return type depends on the value of its parameter: createElement ('div') returns a DivElement, createElement('input') returns an InputElement, etc.

\footnotetext{
${ }^{1}$ http://kotlin.jetbrains.org

${ }^{2}$ http://opalang.org

${ }^{3}$ http://sharpkit.net
} 
Most of the aforementionned languages expose this function by making it return an Element, the least upper bound of the types of all the possible returned values, thus loosing type information and requiring users to explicitly downcast the returned value to its expected, more precise type. Another way to expose this function consists in exposing several functions, each one fixing the value of the initial parameter along with its return type: createDivElement, createInputElement, etc. are parameterless functions returning a DivElement and an InputElement, respectively. This encoding forces to hard-code the name of the to-be created element: it cannot anymore be a parameter. In summary, the first solution is not type safe and the second solution reduces the expressive power of the API.

This paper reviews some common functions of the browser API, identifies the patterns that are difficult to encode in static type systems and shows new ways to encode them in such a way that both type safety and expressive power are preserved. We show that type parameters are sufficient to achieve this goal and that path-dependent types provide an even more convenient encoding of the browser API.

The remainder of the paper is organized as follows. The next section reviews the most common functions of the browser API and how they are typically integrated into statically typed languages. Section 3 shows two ways to improve their integration such that type safety and expressiveness are preserved. Section 4 validates our contribution and discusses its limits. Section 5 discusses some related works and Section 6 concludes.

\section{Background}

This section reviews the most commonly used browser functions and presents the different integration strategies currently used by the statically typed programming languages GWT, Dart, TypeScript, Kotlin, Opa, SharpKit, Haxe, Scala, Idris and Elm.

All these languages support a foreign function interface mechanism, allowing developers to write JavaScript expressions from their programs. Since this mechanism is generally untyped and error prone, most languages (TypeScript, Kotlin, Opa, SharpKit, Haxe, Scala and Elm) support a way to define external typed interfaces. Most of them also expose the browser API this way, as described in the next section.

\subsection{The browser API and its integration in statically typed languages}

The client-side part of the code of a Web application essentially reacts to user events (e.g. mouse clicks), triggers actions and updates the document (DOM) according to their effect. Table 2.1 lists the main functions supported by Web 
browsers according to the Mozilla Developer Network ${ }^{4}$ (we omit the functions that can trivially be encoded in a static type system).

\begin{tabular}{ll}
\hline Name & Description \\
\hline getElementsByTagName(name) & Find elements by their tag name \\
getElementById(id) & Find an element by its id attribute \\
createElement (name) & Create an element \\
target.addEventListener(name, listener) & React to events \\
\hline
\end{tabular}

Table 1. Web browsers main functions that are challenging to encode in a static type system

To illustrate the challenges raised by these functions, we present a simple JavaScript program using them and show how it can be implemented in statically typed programming languages according to the different strategies used to encode these functions. Listing 1 shows the initial JavaScript code of the program. It defines a function slideshow that creates a slide show from an array of image URLs. The function returns an image element displaying the first image of the slide show, and each time a user clicks on it with the mouse left button the next image is displayed.

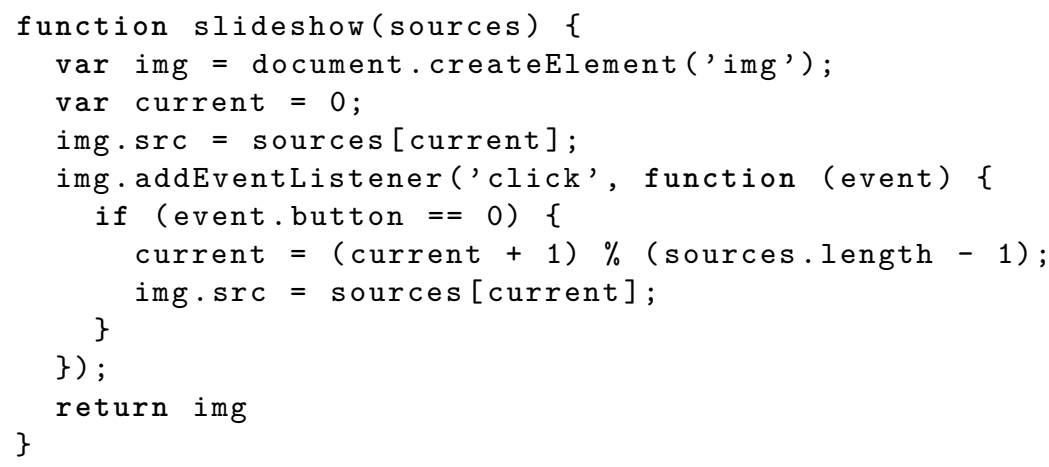

Listing 1. JavaScript function creating a slide show from an array of image URLs

The most common way to encode the DOM API in statically typed languages is to follow the standard interface specifications of HTML [18] and DOM [4].

The main challenge comes from the fact that the parameter types and return types of these functions are often too general. Indeed, functions getElementsByTagName (name), getElementById (id) and createElement (name) can return values of type DivElement or InputElement or any other subtype of Element (their least upper bound). The

\footnotetext{
${ }^{4}$ https://developer.mozilla.org/en-US/docs/DOM/DOM_Reference/Introduction
} 
interface of Element is more general and provides less features than its subtypes. For instance, the ImageElement type (representing images) has a src property that does not exist at the Element level. Similarly, the MouseEvent type has a button property that does not exist at the (more general) Event level, used by the function addEventListener.

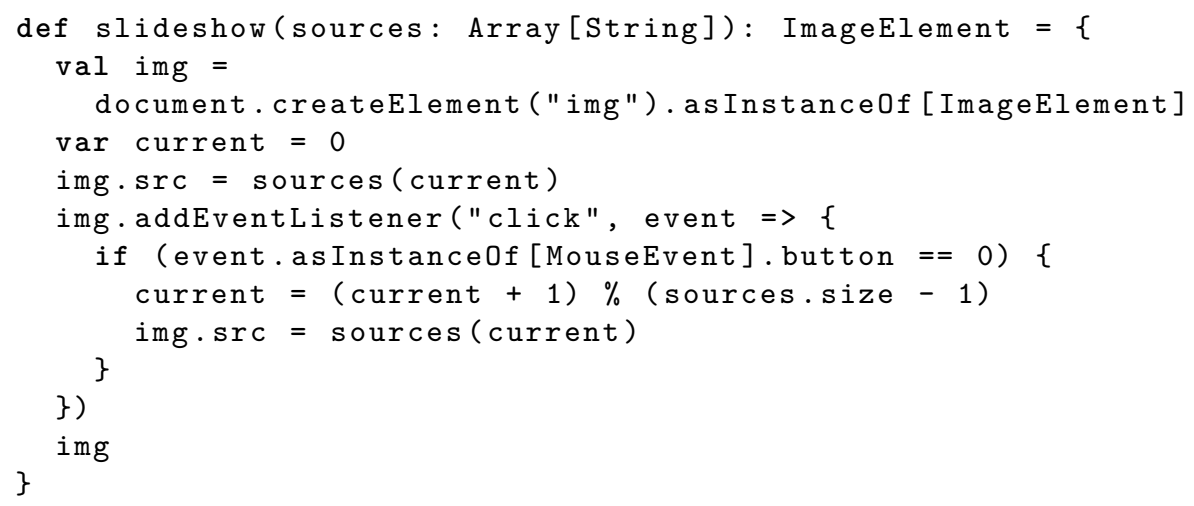

Listing 2. Scala implementation of slideshow using the standard HTML and DOM API

Listing 2 shows a Scala implementation of the slideshow program using an API following the standard specifications of HTML and DOM. The listing contains two type casts, needed to use the src property on the img value and the button property on the event value, respectively.

These type casts make the code more fragile and less convenient to read and write. That's why some statically typed languages attempt to provide an API preserving types as precisely as possible.

Of course, in the case of getElementById(id), the id parameter does not give any clue on the possible type of the searched element, so it is hard to more precisely infer the return type of this function. Hence, most implementations use Element as their return type.

However, in the case of getElementsByTagName (name) and createElement (name), there is exactly one possible return type for each value of the name parameter: e.g. getElementsByTagName ('input') always returns a list of InputElement and createElement ('div') always returns a DivElement. This feature makes it possible to encode these two functions by defining as many parameterless functions as there are possible tag names, where each function fixes the initial name parameter to be one of the possible values and exposes the corresponding specialized return type.

The case of target.addEventListener (name, listener) is a bit different. The name parameter defines the event to listen to while the listener parameter identifies the function to call back each time such an event occurs. Instead of being 
polymorphic in its return type, it is polymorphic in its listener parameter. Nevertheless, a similar property as above holds: there is exactly one possible type for the listener parameter for each value of the name parameter. For instance, a listener of 'click' events is a function taking a MouseEvent parameter, a listener of 'keydown' events is a function taking a KeyboardEvent parameter, and so on. The same pattern as above (defining a set of functions fixing the name parameter value) can be used to encode this function in statically typed languages.

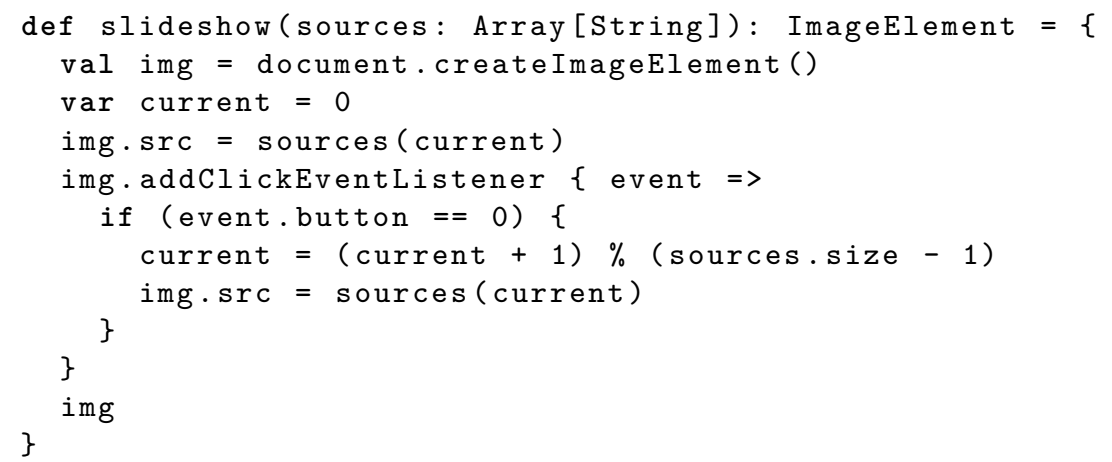

Listing 3. Scala implementation of slideshow using specialized functions

Listing 3 shows what would our slideshow implementation look like using such an encoding. There are two modifications compared to Listing 2: we use document. createImageElement instead of document.createElement, and we use img. addClickEventListener instead of img.addEventListener.

The createImageElement function takes no parameter and returns a value of type ImageElement, and the addClickEventListener function takes as parameter a function that takes a MouseEvent value as parameter, ruling out the need for type casts.

In the case of the addEventListener function we also encountered a slight variation of the encoding, consisting in defining one general function taking one parameter carrying both the information of the event name and the event listener.

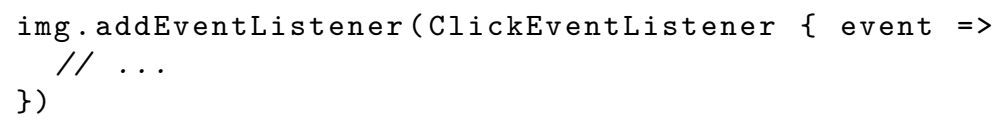

Listing 4. Implementation of slideshow using a general addEventListener function taking one parameter containing both the event name and the even listener 
Listing 4 shows the relevant changes in our program if we use this encoding. The addEventListener function takes one parameter, a ClickEventListener, carrying both the name of the event and the event listener code.

Most of the studied languages expose the browser API following the standard specification, but some of them (GWT, Dart, HaXe and Elm) define a modified API getting rid of (or at least reducing) the need for downcasting, following the approaches described above.

\subsection{Limitations of existing encoding approaches}

We distinguished three approaches to integrate the challenging parts of the browser API into statically typed languages. This section shows that each approach favours either type safety or expressive power but none provides both type safety and the same expressive power as the native browser API. We indeed consider that an API requiring users to do type casts is not type safe, while an API making it impossible to implement a function that can readily be implemented using the native API gives less expressive power to the programmer.

The first approach, consisting in using the least upper bound of all the possible types has the same expressive power as the native browser API, but is not type safe because it sometimes requires developers to explicitly downcast values to their expected specialized type.

The second approach, consisting in defining as many functions as there are possible return types of the encoded function, is type safe but leads to a less general API: each function fixes a parameter value of the encoded function, hence being less general. The limits of this approach are better illustrated when one tries to combine several functions. Consider for instance Listing 5 defining a JavaScript function findAndListenTo that both finds elements and registers an event listener when a given event occurs on them. Note that the event listener is passed both the event and the element: its type depends on both the tag name and the event name. This function cannot be implemented if the general functions getElementsByTagName and addEventListener are not available. The best that could be done would be to create one function for each combination of tag name and event name, leading to an explosion of the number of functions to implement. Thus, this approach gives less expressive power than the native browser API. Moreover, we find that defining many functions for the same task (creating a DOM element or listening to an event) clutters the API documentation: functions serving other purposes are hidden by these same-purpose-functions.

The third approach, consisting in combining two parameters into one parameter carrying all the required information, is type safe too, but reduces the expressive power because it forbids developers to partially apply the function by supplying only one parameter. Consider for instance Listing 6 that defines a function observe partially applying the addEventListener function ${ }^{5}$. Such a function cannot be implemented with this approach because the name of the

\footnotetext{
5 The code of this function has been taken (and simplified) from the existing functional reactive programming libraries Rx.js [14] and Bacon.js (http://baconjs.github.io/).
} 


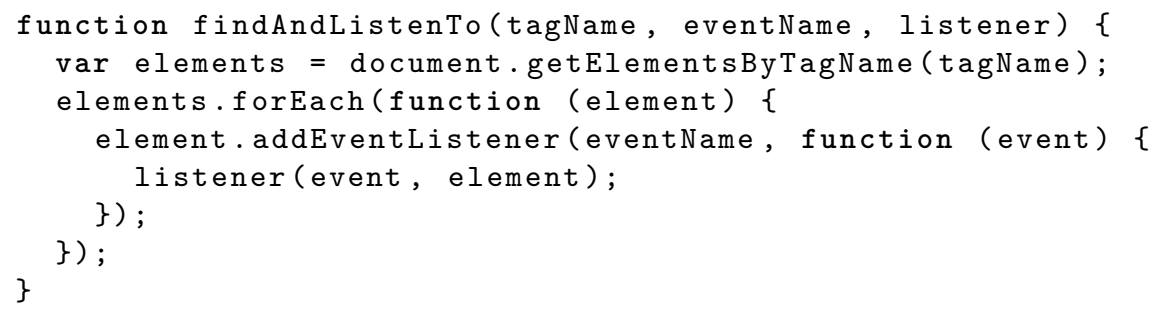

Listing 5. Combination of use of getElementsByTagName and addEventListener

event and the code of the listener cannot be decoupled. Thus, this one gives less expressive power than the native browser API.

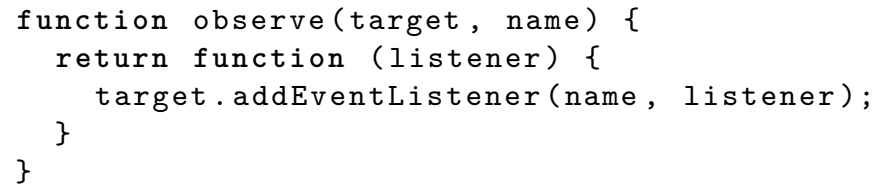

Listing 6. Partial application of addEventListener parameters

In summary, the current integration of the browser API by statically typed languages compiling to JavaScript is either not type safe or not as expressive as the underlying JavaScript API. Indeed, we showed that our simple slideshow program requires type casts if the browser API is exposed according to the standard specification. We are able to get rid of type casts on this program by using modified browser APIs, but we presented two functions that we were not able to implement using these APIs, showing that they give less expressive power than the native API.

This article aims to answer the following questions: is it possible to expose the browser API in statically typed languages in a way that both reduces the need for type casts and preserves the same expressive power? What typing mechanisms do we need to achieve this? Would it be convenient to be used by end developers?

\section{Contribution}

In this section we show how we can encode the challenging main functions of the DOM API in a type safe way while keeping the same expressive power.

The listings in this paper use the Scala language, though our first solution could be implemented in any language with basic type parameters support, such 
as Java's generics ${ }^{6}$. Our second solution is an improvement over the first one, using path-dependent types.

\subsection{Parametric Polymorphism}

In all the cases where a type $\mathrm{T}$ involved in a function depends on the value of a parameter $\mathrm{p}$ of this function (all the aforementionned functions of the DOM API are in this case), we can encode this relationship in the type system using type parameters as follows:

1. Define a parameterized class $\mathrm{P}[\mathrm{U}]$

2. Set the type of $p$ to $P[U]$

3. Use type $\mathrm{U}$ instead of type $\mathrm{T}$

4. Define as many values of type $\mathrm{P}[\mathrm{U}]$ as there are possible values for $\mathrm{p}$, each one fixing its $\mathrm{U}$ type parameter to the corresponding more precise type

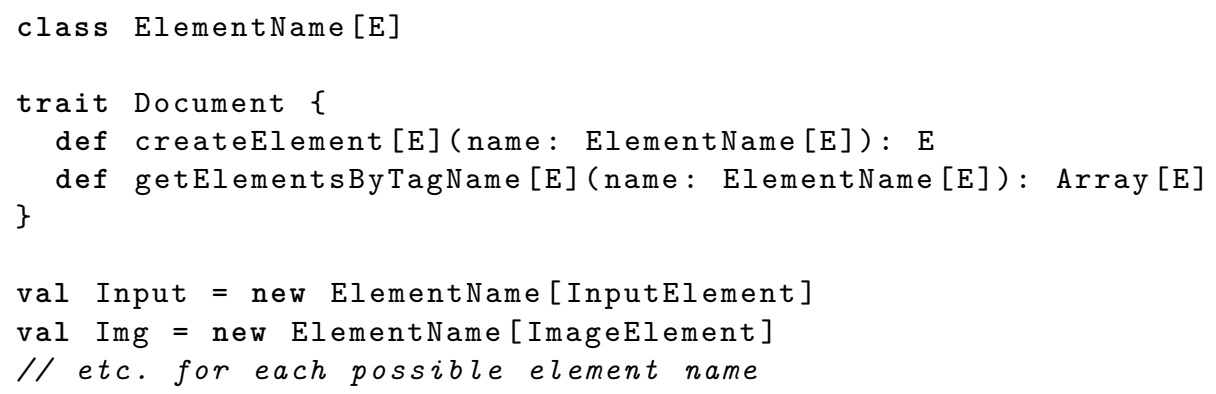

Listing 7. Encoding of the createElement function using type parameters

Listing 7 shows this approach applied to the createElement and getElementsByTagName functions which return type depends on their name parameter value: a type ElementName $[\mathrm{E}]$ has been created, the type of the name parameter has been set to ElementName[E] instead of String, and the return type of the function is E instead of Element (or Array[E] instead of Array[Element], in the case of getElementsByTagName). The ElementName [E] type encodes the relationship between the name of an element and the type of this element ${ }^{7}$. For instance, we created a value Input of type ElementName [InputElement].

Listing 8 shows the encoding of the addEventListener function. The EventName [E] type represents the name of an event which type is E. For instance, Click is a

${ }^{6}$ For a lack of space, we do not present them here but all Java versions of all the Scala listings (excepted those using type members) are available online at http://github.com/js-scala/js-scala/wiki/ICWE'14

7 The type parameter $\mathrm{E}$ is also called a phantom type [12] because ElementName values never hold a $E$ value 


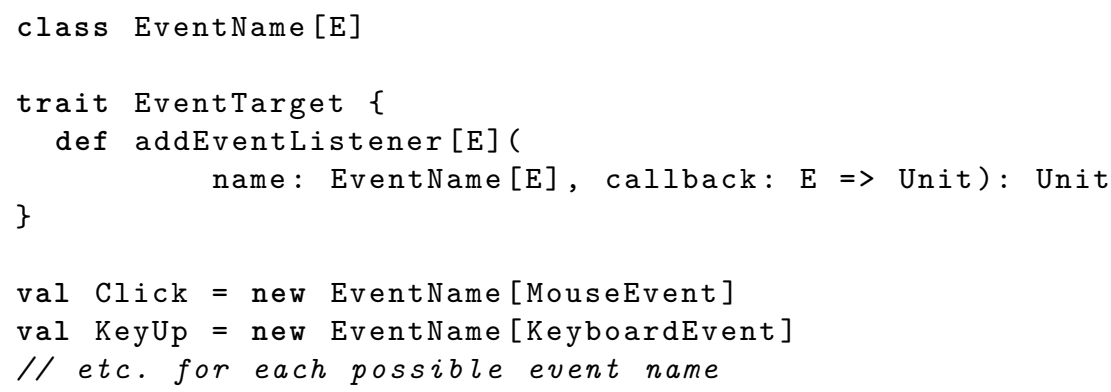

Listing 8. Encoding of the addEventListener function using type parameters

value of type EventName [MouseEvent]: when a user adds an event listener to the Click event, it fixes to MouseEvent the type parameter $\mathrm{E}$ of the callback function passed to addEventListener.

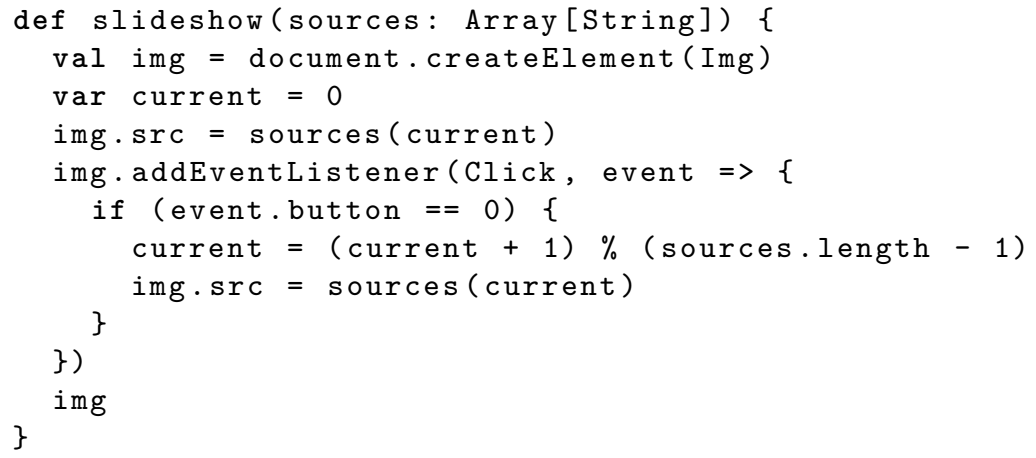

Listing 9. Scala implementation of the slideshow function using generics

Listing 9 illustrates the usage of such an encoding by implementing our slideshow program presented in the introduction. Passing the Img value as a parameter to the createElement function fixes its E type parameter to ImageElement so the returned value has the most possible precise type and the src property can be used on it. Similarly, passing the Click value to the addEventListener function fixes its E type parameter to MouseEvent, so the event listener has the most possible precise type and the button property can be used on the event parameter.

It is worth noting that this code is actually exactly the same as in Listing 2 excepted that type casts are not anymore required because the browser API is exposed in a way that preserves enough type information. Our way to encode the browser API is more type safe, but is it as expressive as the native API? 


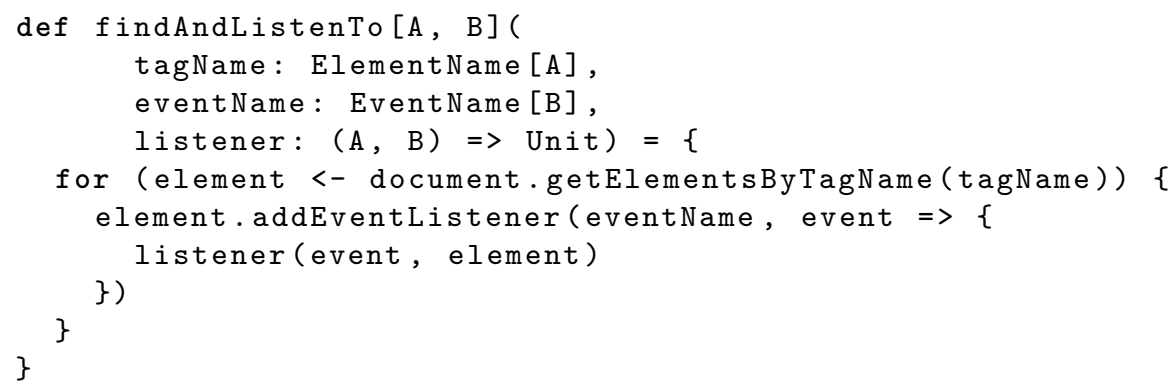

Listing 10. Combination of getElementsByTagName and addEventListener functions encoded using type parameters

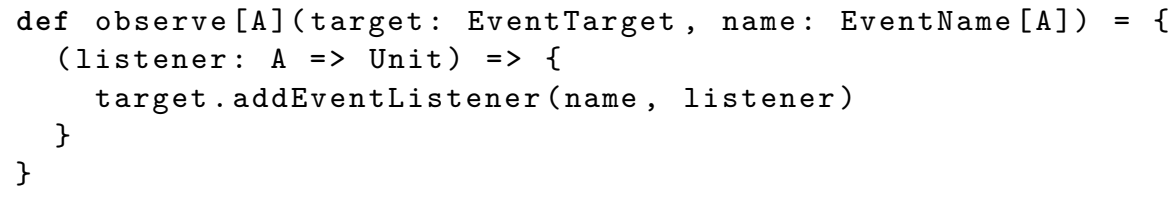

Listing 11. Partial application of addEventListener encoded with type parameters

Listings 10 and 11 show how the challenging functions of Section 2.2, findAndListenTo and observe, can be implemented with our encoding. They are basically a direct translation from JavaScript syntax to Scala syntax, with additional type annotations.

In summary, our encoding is type safe and gives as much expressive power as the native API since it is possible to implement exactly the same functions as we are able to implement in plain JavaScript.

However, every function taking an element name or an event name as parameter has its type signature cluttered with phantom types (extra type parameters): the observe function takes a phantom type parameter A and the findAndListenTo function takes two phantom type parameters, A and B. These extra type parameters are redundant with their corresponding value parameters and they make type signatures harder to read and reason about.

\subsection{Path-Dependent Types}

This section shows how we can remove the extra type parameters needed in the previous section by using path-dependent types [16]. Essentially, the idea is to model type parameters using type members, as suggested in [17].

Programming languages generally support two means of abstraction: parameterization and abstract members. For instance Java supports parameterization for values (method parameters) and types (generics), and member abstraction for values (abstract methods). Scala also supports member abstraction for types through type members $[5,16]$. An abstract type member of a class is an inner 
abstract type that can be used to qualify values. Subclasses can implement and override their methods, and similarly they can define or refine their type members. A concrete subclass must provide a concrete implementation of its type members. Outside of the class, type members can be referred to using a type selection on an instance of the class: the type designator p.C refers to the $\mathrm{C}$ type member of the value $\mathrm{p}$ and expands to the $\mathrm{C}$ type member implementation of the singleton type of $\mathrm{p}$.

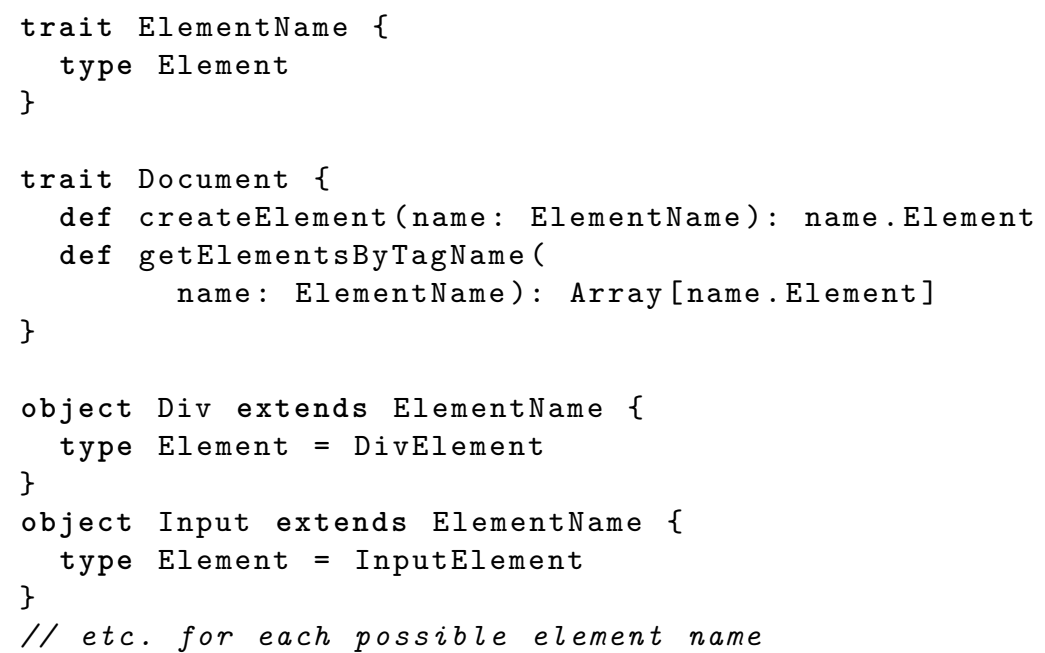

Listing 12. Encoding of createElement using path-dependent types

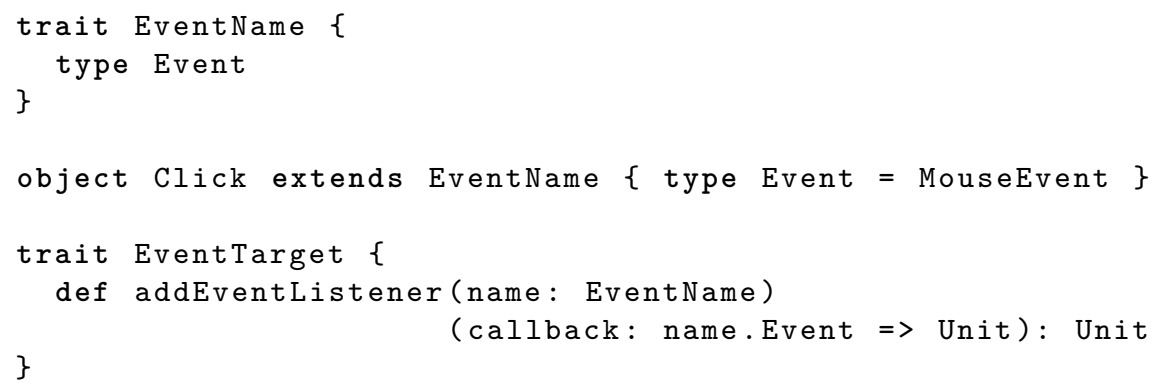

Listing 13. Encoding of addEventListener using path-dependent types

Listings 12 and 13 show an encoding of createElement, getElementsByTagName and addEventListener in Scala using type members. Now, the ElementName type 
has no type parameter but a type member Element. The return type of the createElement function is name.Element: it refers to the Element type member of its name parameter. The Div and Input values illustrate how their corresponding element type is fixed: if one writes createElement (Input), the return type is the Element type member of the Input value, namely InputElement. The same idea applies to EventName and addEventListener: the name of the event fixes the type of the callback.

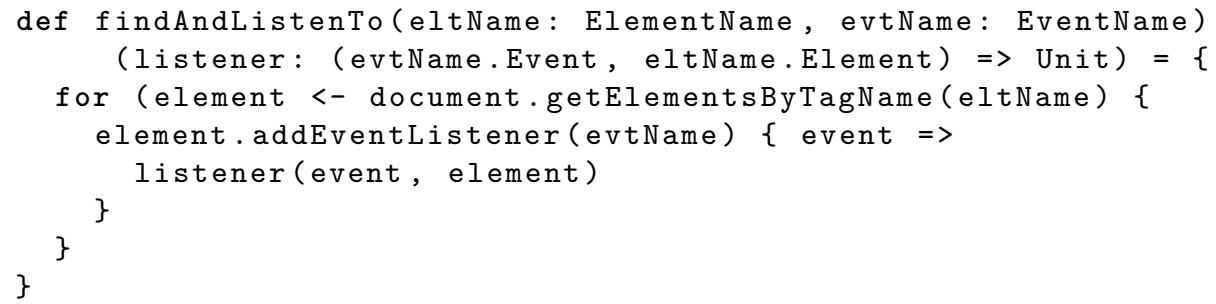

Listing 14. Combination of getElementsByTagName and addEventListener using path-dependent types

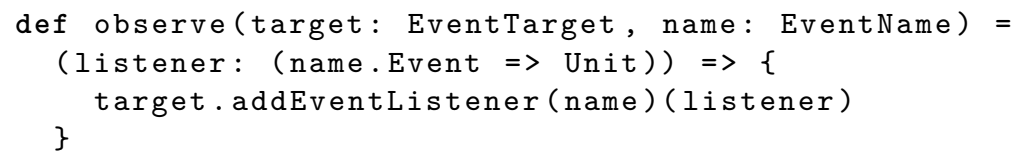

Listing 15. Partial application of addEventListener using path-dependent types

The implementation of the slideshow function with this encoding is exactly the same as with the previous approach using generics. However, functions findAndListenTo and observe can be implemented more straightforwardly, as shown by listings 14 and 15, respectively.

With this encoding, the functions using event names or element names are not anymore cluttered with phantom types, and type safety is still preserved.

\section{Validation}

\subsection{Implementation in js-scala}

We implemented our encoding in js-scala [11], a Scala library providing composable JavaScript code generators ${ }^{8}$. On top of that we implemented various

\footnotetext{
${ }_{8}$ Source code is available at http://github.com/js-scala
} 
samples, including non trivial ones like a realtime chat application and a poll application.

We have shown in this paper that our encoding leverages types as precisely as possible (our slideshow program is free of type casts) while being expressive enough to implement the challenging findAndListenTo and observe functions that were impossible to implement with other approaches.

\subsection{API clarity}

We mentioned in the background section that a common drawback of existing approaches to bring more type safety was the multiplication of functions having the same purpose, making the API documentation harder to read.

Our encoding preserves a one to one mapping with browser API functions whereas existing approaches often have more than 30 functions for a same purpose. For instance, the createElement function is mapped by 31 specialized functions in GWT and 62 in Dart, the addEventListener is mapped by 32 specialized functions in GWT and 49 in Dart.

\subsection{Convenience for end developers}

Statically typed languages are often criticized for the verbosity of the information they add compared to dynamically typed languages [15]. In our case, what is the price to pay to get accurate type ascriptions?

The first encoding, using type parameters, can be implemented in most programming languages because it only requires a basic support of type parameters (for instance Java, Dart, TypeScript, Kotlin, HaXe, Opa, Idris and Elm can implement it). However this encoding leads to cluttered type signatures and forces functions parameterized by event or element names to also take phantom type parameters.

However, the second encoding, using type members, leads to type signatures that are not more verbose than those of the standard specifications of the HTML and DOM APIs, so we argue that there is no price to pay. However, this encoding can only be implemented in language supporting type members or dependent types (Scala and Idris).

\subsection{Limitations}

Our encodings only work with cases where a polymorphic type can be fixed by a value. In our examples, the only one that is not in this case is getElementById. Therefore we are not able to type this function more accurately (achieving this would require to support the DOM tree itself in the type system as in [13]).

Our solution is actually slightly less expressive than the JavaScript API: indeed, the value representing the name of an event or an element is not anymore a String, so it cannot anymore be the result of a String manipulation, like e.g. a concatenation. Fortunately, this case is uncommon. 


\section{Related Works}

The idea of using dependent types to type JavaScript has already been explored by Ravi Chugh et. al. [3]. They showed how to make a subset of JavaScript statically typed using a dependent type system. However, their solution requires complex and verbose type annotations to be written by developers.

Sebastien Doreane proposed a way to integrate JavaScript APIs in Scala [7]. His approach allows developers to seamlessly use JavaScript APIs from statically typed Scala code. However, his work does not expose types as precise as ours (e.g. in their encoding the return type of createElement is always Element).

TypeScript supports overloading on constant values: the type of the expression createElement ("div") is statically resolved to DivElement by the constant parameter value "div". This solution is type safe, as expressive and as easy to learn as the native API because its functions have a one to one mapping. However, this kind of overloading has limited applicability because overload resolution requires parameters to be constant values: indeed, the findAndListenTo function would be weakly typed with this approach.

\section{Conclusion}

Having a statically typed programming language compiling to JavaScript is not enough to leverage static typing in Web applications. The native browser API has to be exposed in a statically typed way, but this is not an easy task.

We presented two ways to encode dynamically typed browser functions in mainstream statically typed languages like Java and Scala, using type parameters or path-dependent types. Our encodings give more type safety than existing solutions while keeping the same expressive power as the native API.

We feel that parametric polymorphism and, even more, dependent types are precious type system features for languages aiming to bring static typing to Web applications.

Acknowledgements The authors would like to thank Thomas Degueule.

\section{References}

1. Edwin Brady. Idris, a general-purpose dependently typed programming language: Design and implementation. Journal of Functional Programming, 23(05):552-593, 2013.

2. N. Cannasse. Using haxe. The Essential Guide to Open Source Flash Development, pages 227-244, 2008.

3. Ravi Chugh, David Herman, and Ranjit Jhala. Dependent types for javascript. SIGPLAN Not., 47(10):587-606, October 2012.

4. W3C-World Wide Web Consortium et al. Document object model (dom) level 3 core specification. W3C recommendation, 2004. 
5. Vincent Cremet, François Garillot, Sergueï Lenglet, and Martin Odersky. A core calculus for scala type checking. In Mathematical Foundations of Computer Science 2006, pages 1-23. Springer, 2006.

6. Evan Czaplicki. Elm: Concurrent frp for functional guis, 2012.

7. Sébastien Doeraene. Scala.js: Type-Directed Interoperability with Dynamically Typed Languages. Technical report, 2013.

8. Steve Fenton. Typescript for javascript programmers. 2012.

9. R. Griffith. The dart programming language for non-programmers-overview. 2011.

10. Federico Kereki. Web 2.0 development with the Google web toolkit. Linux J., 2009(178), February 2009.

11. Grzegorz Kossakowski, Nada Amin, Tiark Rompf, and Martin Odersky. JavaScript as an Embedded DSL. In James Noble, editor, ECOOP 2012 - Object-Oriented Programming, volume 7313 of Lecture Notes in Computer Science, pages 409-434, Berlin, Heidelberg, 2012. Springer Berlin Heidelberg.

12. Daan Leijen and Erik Meijer. Domain specific embedded compilers. In $A C M$ SIGPLAN Notices, volume 35, pages 109-122. ACM, 1999.

13. BenjaminS. Lerner, Liam Elberty, Jincheng Li, and Shriram Krishnamurthi. Combining Form and Function: Static Types for JQuery Programs. In Giuseppe Castagna, editor, ECOOP 2013 - Object-Oriented Programming, volume 7920 of Lecture Notes in Computer Science, pages 79-103. Springer Berlin Heidelberg, 2013.

14. Jesse Liberty and Paul Betts. Reactive extensions for javascript. In Programming Reactive Extensions and LINQ, pages 111-124. Springer, 2011.

15. Erik Meijer and Peter Drayton. Static typing where possible, dynamic typing when needed: The end of the cold war between programming languages.

16. Martin Odersky, Vincent Cremet, Christine Röckl, and Matthias Zenger. A nominal theory of objects with dependent types. In Luca Cardelli, editor, ECOOP 2003 - Object-Oriented Programming, volume 2743 of Lecture Notes in Computer Science, pages 201-224. Springer Berlin Heidelberg, 2003.

17. Martin Odersky and Matthias Zenger. Scalable component abstractions. In $A C M$ SIGPLAN Notices, volume 40, pages 41-57. ACM, 2005.

18. Dave Raggett, Arnaud Le Hors, Ian Jacobs, et al. Html 4.01 specification. W3C recommendation, 24, 1999. 